\title{
Personality and Emotion
}

\author{
William Revelle \\ Department of Psychology \\ Northwestern University
}

\author{
Klaus R. Scherer \\ Swiss Center for Affective Sciences \\ University of Geneva
}

Personality is the coherent patterning of affect, behavior, cognition, and desires (goals) over time and space. Just as a full blown emotion represents an integration of feeling, action, appraisal and wants at a particular time and location so does personality represent integration over time and space of these components (Ortony et al., 2005). A helpful analogy is to consider that personality is to emotion as climate is to weather. That is, what one expects is personality, what one observes at any particular moment is emotion.

To understand the personality-affect link it is necessary to consider the ways in which personality may be described. Since Theophrastus' discussion of characters and Galen's theory of temperament (Stelmack \& Stalikas, 1991), dimensional models of individual differences in personality have consistently identified three (the Giant Three, e.g., Eysenck \& Eysenck (1985)) to five (the Big Five, e.g., Digman (1990)) broad dimensions of personality. Two of these dimensions, in particular, Extraversion (E) and Neuroticism (N, sometimes referred to by the other end of the dimension as Emotional Stability) have been associated with individual differences in affective level and environmental responsivity (Corr, 2008; Revelle, 1995).

Ever since antiquity, starting with Galen's classification of the four different humors, it has been assumed that individuals differ in their predisposition to experience certain emotions. Extrapolating from animal studies, $\mathrm{E}$ and $\mathrm{N}$ have been associated with the Behavioral Activation System (BAS) and Behavioral Inhibition System (BIS) respectively, while distinctions between trait fear and trait anxiety have been associated with the Fight/Freeze/Flight System (FFFS) (Corr, 2008; Gray \& McNaughton, 2000). Indeed, the basic assumptions of Reinforcement Sensitivity Theory (Corr, 2008), perhaps better labeled as Three Systems Theory, are that the stable personality traits reflect individual differences in reactivity to emotional and affectively va- lenced environmental cues.

Descriptively, there is much literature on hysteric, neurotic, or anhedonic personalities (Kellerman, 1990), or, in more recent terminology, on trait anger, trait anxiety, or trait positive-negative affect (Spielberger et al., 1999; Tellegen et al., 1999) $<$ cross-ref Affective style $>$. These trait differences in emotionality increase the odds of experiencing trait-congruent emotions. In other words, individuals high on trait anxiety run an increased risk of experiencing anxiety bouts, individuals high on trait anger get irritated more often, and so forth.

Thus, in a quasi-representative survey of everyday emotion experiences Scherer et al. (2004) showed that the emotionality dispositions may significantly increase the risk to experience certain emotions. Concretely, the more frequently respondents habitually experienced a particular kind of emotion (trait emotionality), the more likely they experienced an exemplar of that emotion category yesterday. Thus, respondents high on trait anxiety were almost three times as likely to have experienced anxiety yesterday compared to those who are low on this trait. In the case of trait sadness and trait despair, the likelihood is about two times higher. Respondents high on trait irritation are about 1.5 times more likely to have experienced anger yesterday. Similarly, respondents reporting frequent habitual pleasure, surprise, or pride experiences are also 1.5 times more likely to have experienced joy or happiness. Because some emotions occur less frequently than expected for respondents with certain habitual emotion dispositions, some types of trait emotionality might inoculate, or shield, against particular emotions. The results seem to indicate that trait pleasure may reduce the risk of despair, and that trait surprise may reduce the risk of anxiety.

These results do not just reflect common responses to questionnaires, but rather reflect basic neural processes. Using functional brain mapping (e.g., fMRI), trait extraversion and neuroticism were 
associated with differential activation to rewarding or positive slides (extraversion) and to threat cues (neuroticism). Extraversion was correlated with amount of activation in widely distributed brain regions (amygdala, caudate, medio-frontal gyrus, right fusiform gyrus) in response to positively valenced slides but unrelated to activation to negatively valenced slides. Neuroticism, on the other hand, was correlated with amount of activation to negatively valenced slides, but unrelated to activation to positively valenced slides (Canli, 2004).

Taken together, these results seem to strongly confirm the notion of habitual or trait emotionality; that is, an individual difference variable consisting of a disposition to experience certain types of emotions more frequently than other people. While this notion is widely accepted for trait anxiety and trait anger (Spielberger et al., 1999), as well as trait positive affect (Tellegen et al., 1999), other types of trait emotionality have rarely been investigated. Nor is the relationship of affect to the other three of the "Big 5" as well established.

To some, extraversion is just trait positive affect and neuroticism is just trait negative affect. Although trait extraversion is associated with trait positive affect in many cultures (Lucas \& Baird, 2004) and has been proposed to have the tendency to experience positive affect at its core (Watson \& Clark, 1997), extraversion is more than positive affect, for it also represents differences in behavior, cognition, and desires (Wilt \& Revelle, 2008). Extraversion is related to general activation and behavioral approach as well as ways of categorizing words in terms of their semantic associates (Rogers \& Revelle, 1998). Similarly, neuroticism is not just negative affect, for it has cognitive and behavioral components as well. Other non-affective components of extraversion and neuroticism include differences in desires, with extraversion associated with a need for social contact, power and status and neuroticism associated with needs for acceptance, tranquility, order, vengenace, and savings (Olson \& Weber, 2004).

One of the issues that remains difficult to assess is the origin of such affect dispositions. While clearly sharing a large genetic component (Bouchard, 2004) some of these dispositions may in fact be already prepared at birth or early childhood (Durbin et al., 2005) (<cross-ref Genetics, Temperament $>$ ), others may develop through learn- ing and socialization, often in interaction with innate dispositions (Caspi et al., 2005). Of course, many other individual factors may also play a role.

Recently, attribution and appraisal theorists have suggested that specific styles of causal attribution $(<$ cross-ref attributional styles $>$ ) or appraisal styles $(<$ cross-ref appraisal styles $>$ ) in a very general sense can lead certain persons to be more or less prone to experience certain types of emotions because of differences in goals, values, and coping potential (Reekum \& Scherer, 1997). This is particularly salient, when a person demonstrates an appraisal bias which may lead to dysfunctional and unrealistic appraisals and in consequence, to maladaptive emotions or even emotional disturbances (Kaiser \& Scherer, 1998; Roseman \& Kaiser, 2001). An appraisal bias would be exhibited if a person has a tendency to always over- or underestimate the responsibility of self or another person for a failure experience, to systematically, irrespective of circumstances or over- or underestimate his or her coping potential. Like the question of the origin of affect dispositions, it will be important to examine to what extent these biases are effects of individual learning or socialization careers or whether cultural or social group factors may play a major role in sensitizing individuals to certain appraisal possibilities or to privilege certain types of appraisal style.

Although representing different research traditions, normal and abnormal personality may be integrated into a common framework with respect to individual differences in affective processes (Krueger $\&$ Tackett, 2006; Markon et al., 2005). Extreme levels of activation of the BIS, BAS, or FFFS have been used as explanations of many of the personality disorders, particularly those associated with uninhibited behaviors, "externalizing" disorders, overly inhibited anxious behaviors and "internalizing" disorders. The search for a common core to normal and abnormal personality has emphasized the study of affective and cognitive deficits in psychopathology as they relate to multiples levels of processing, from the reactive, to the routine, to the reflective (Ortony et al., 2005; Reekum \& Scherer, 1997).

In that personality represents the integration over time of feelings, actions, thoughts and desires, theoretical developments in personality benefit from a greater understanding of emotional processes. At the same time, research in emotion can take advantage of individual differences in sensitivities to situ- 
ational cues and predispositions to emotional states. The questions of why some people become angry, while others become frightened or depressed in response to threats, and why some become elated while others seem unaffected when given rewards will be better understood by jointly studying the problem of long term coherence (personality) with short term fluctuations in affect, behavior, cognition and desire (emotion).

\section{References}

Bouchard, T. J. (2004). Genetic influence on human psychological traits: A survey. Current Directions in Psychological Science, 13(4), 148-151.

Canli, T. (2004). Functional brain mapping of extraversion and neuroticism: Learning from individual differences in emotion processing. Journal of Personality, 72(6), 1105-1132.

Caspi, A., Roberts, B. W., \& Shiner, R. L. (2005). Personality development: Stability and change. Annual Review of Psychology, 56, 453-484.

Corr, P. J.(2008). The reinforcement sensitivity theory. In P. J. Corr (Ed.), The reinforcement sensitivity theory of personality. Cambridge: Cambridge University Press.

Digman, J. M. (1990). Personality structure: Emergence of the five-factor model. Annual Review of Psychology., 41, 417-440.

Durbin, C., Klein, D. N., Hayden, E. P., Buckley, M. E., \& Moerk, K. C. (2005). Temperamental emotionality in preschoolers and parental mood disorders. Journal of Abnormal Psychology, 114(1), 28-37.

Eysenck, H. J., \& Eysenck, M. W.(1985). Personality and individual differences: a natural science approach. New York: Plenum Press.

Gray, J. A., \& McNaughton, N. (2000). The neuropsychology of anxiety: An enquiry into the functions of the septo-hippocampal system (2nd ed.). Oxford: Oxford University Press.

Kaiser, S., \& Scherer, K. R.(1998). Models of "normal" emotions applied to facial and vocal expression in clinical disorders. In J. Flack William F. \& J. D. Laird (Eds.), Emotions in psychopathology:
Theory and research (p. 81-98). Oxford University Press.

Kellerman, H.(1990). Emotion and the organization of primary process. In R. Plutchik \& H. Kellerman (Eds.), Emotion, psychopathology, and psychotherapy (p. 89-113). San Diego, CA: Academic Press.

Krueger, R. F., \& Tackett, J. L. (2006). Personality and psychopathology. Personality and psychopathology. xii, 402 pp. New York, NY: Guilford Press.

Lucas, R. E., \& Baird, B. M. (2004). Extraversion and emotional reactivity. Journal of Personality and Social Psychology, 86(3), 473-485.

Markon, K. E., Krueger, R. F., \& Watson, D.(2005). Delineating the structure of normal and abnormal personality: An integrative hierarchical approach. Journal of Personality and Social Psychology, 88(1), 139-157.

Olson, K. R., \& Weber, D. A. (2004). Relations between big five traits and fundamental motives. Psychological Reports, 95(3), 795-802.

Ortony, A., Norman, D. A., \& Revelle, W. (2005). Effective functioning: A three level model of affect, motivation, cognition, and behavior. In J. Fellous \& M. Arbib (Eds.), Who needs emotions? The brain meets the machine. (p. 173202). New York: Oxford Univeristy Press.

Reekum, C. M. van, \& Scherer, K. R.(1997). Levels of processing for emotion-antecedent appraisal. In G. Matthews (Ed.), Cognitive science perspectives on personality and emotion (p. 259-300). Amsterdam: Elsevier Science.

Revelle, W. (1995). Personality processes. Annual Review of Psychology, 46.

Rogers, G. M., \& Revelle, W. (1998). Personality, mood, and the evaluation of affective and neutral word pairs. Journal of Personality and Social Psychology, 74(6), 1592-1605.

Roseman, I. J., \& Kaiser, S.(2001). Applications of appraisal theory to understanding, diagnosis, and treating emotional pathology. In K. R. Scherer, 
A. Schorr, \& T. Johnstone (Eds.), Appraisal processes in emotion: Theory, methods, research ( $\mathrm{p}$. 249-267). New York, NY: Oxford University Press.

Scherer, K. R., Wranik, T., Sangsue, J., Tran, V., \& Scherer, U. (2004). Emotions in everyday life: Probability of occurrence, risk factors, appraisal and reaction pattern. Social Science Information, 43(4), 499-570.

Spielberger, C. D., Sydeman, S. J., Owen, A. E., \& Marsh, B. J.(1999). Measuring anxiety and anger with the state-trait anxiety inventory (stai) and the state-trait anger expression inventory (staxi). In M. E. Maruish (Ed.), The use of psychological testing for treatment planning and outcomes assessment (2nd ed.) (p. 993-1021). Mahwah, NJ: Lawrence Erlbaum Associates Publishers.
Stelmack, R. M., \& Stalikas, A. (1991). Galen and the humour theory of temperament. Personality and Individual Differences, 12(3), 255-263.

Tellegen, A., Watson, D., \& Clark, L. A. (1999). On the dimensional and hierarchical structure of affect. Psychological Science, 10(4), 297-303.

Watson, D., \& Clark, L. A.(1997). Extraversion and its positive emotional core. In R. Hogan, J. Johnson, \& S. Briggs (Eds.), Handbook of personality psychology (p. 767-793). San Diego, CA: Academic Press.

Wilt, J., \& Revelle, W. (2008). Extraversion and emotional reactivity. In M. Leary \& R. H. Hoyle (Eds.), (in press) handbook of individual differences in social behavior. New York, N.Y.: Guilford Press. 